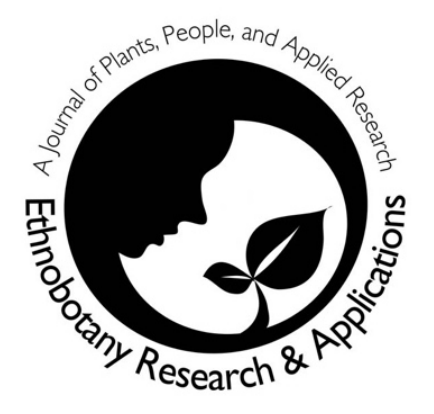

\title{
Los conocimientos de estudios etnobiológicos se ajustan a sistemas de equilibrio
}

\author{
Mónica Moraes R.
}

\section{Editorial}

"Los pueblos indígenas y las comunidades locales poseen un conocimiento detallado sobre la biodiversidad y tendencias de los ecosistemas. Este conocimiento se forma a través de la dependencia directa de sus ecosistemas locales y de las observaciones e interpretaciones del cambio generadas y transmitidas a lo largo de muchas generaciones aún adaptadas y enriquecidas con el tiempo. Los pueblos indígenas y las comunidades locales de todo el mundo a menudo viven en áreas remotas, interactúan con la naturaleza y administran recursos que contribuyen a la sociedad en general. También sufren directamente las presiones de la expansión de las fronteras agrícolas y la producción de productos básicos, como la minería, la tala y la energía. A menudo están mejor ubicados que los científicos para proporcionar información detallada sobre la biodiversidad local y el cambio ambiental, y son importantes contribuyentes a la gestión de la biodiversidad desde el nivel local hasta el global". Panel Intergubernamental sobre Biodiversidad y Servicios de los Ecosistemas" (IPBES)

El desempeño del trabajo de campo de los estudios etnobiológicos con comunidades humanas ha cubierto trayectorias muy contrastantes y una cronología que alcanza el presente, que se nutre de más experiencias en redes de investigación y, en consecuencia, a un nivel de colaboración más cercano. En general, se están realizando esfuerzos para revertir las antiguas prácticas de "extracción de conocimiento exhaustivo", que favorecieron principalmente a una de las partes, una larga lista de estudios descontinuados, ofertas no realizadas por personas e instituciones, entre otras. En esta contribución resumiré algunas percepciones derivadas de los estudios etnobiológicos recientes realizados en Bolivia, en respuesta a los desafíos regionales y al consenso de los científicos que han promovido un contenido cada vez más fortificado.
Varias organizaciones han discutido sobre varios escenarios en los que la ciencia, la naturaleza y las comunidades humanas convergen. Organizaciones internacionales, por ejemplo, regulaciones temáticas en la Convención de las Naciones Unidas sobre la Diversidad Biológica, la Determinación de los Pueblos, el Protocolo de Nagoya y el funcionamiento de las redes de cooperación, como la Sociedad Latinoamericana de Etnobiología (SOLAE) han convocado a varios actores. En general se procura ajustar e incluir recomendaciones para aplicar mejores procedimientos. Todas las directrices, conceptos y recomendaciones han tenido puntos de encuentro que, por supuesto, favorecen a los estudios en colaboración. Un ejemplo a nivel regional fue el proyecto "Acceso y participación en los beneficios en América Latina y el Caribe: un diálogo entre la ciencia y la política en materia de investigación académica" realizado durante 2013 por Diversitas, la Oficina Regional para América Latina y el Caribe de la Organización Internacional. Consejo para la Ciencia (ICSU-ROLAC), la Academia Suiza de Ciencias (SCNAT) y la Unión Internacional de Ciencias Biológicas (IUBS).

\section{Correspondence}

\section{Mónica Moraes R.}

Herbario Nacional de Bolivia, Instituto de Ecología, Facultad de Ciencias Puras y Naturales, Universidad Mayor de San Andrés, Calle 27 Cota Cota s / $\mathrm{n}$ campus universitario La Paz, Bolivia

mmoraes@fcpn.edu.bo

\section{Ethnobotany Research \& Applications} 17:1 (2018)

El objetivo fue recopilar información sobre los elementos destacados del acceso y la distribución de beneficios para la investigación científica en la región de América Latina y el Caribe. Durante los 
talleres organizados, ambos componentes, el académico y el político en instancias gubernamentales tuvieron lugar y discutieron sobre diferentes temas; estudios de caso de ocho países fueron incluidos en el documento compilado. Como parte del informe final, se resumieron sustancialmente en la implementación institucional del sistema de acceso y distribución de beneficios, así como las condiciones y oportunidades favorables previas al proceso de investigación.

Además, el Panel Intergubernamental de Naciones Unidas sobre Biodiversidad y Servicios de los Ecosistemas (IPBES, por sus siglas en inglés), que se creó en abril de 2012, tiene el mandato de evaluar el estado de la biodiversidad y los ecosistemas del planeta y proporcionar información científica accesible a los responsables políticos. El conocimiento indígena y local (ILK, por sus siglas en inglés) tiene el propósito de respaldar el logro del producto 1(c) del programa de trabajo, en relación con los procedimientos y los enfoques para trabajar con los sistemas de conocimiento locales e indígenas. Entre sus responsabilidades se encuentran las siguientes (https://www.ipbes.net/indigenous-local-knowledgemandate): Supervisar el desarrollo de procedimientos y enfoques para trabajar con sistemas de conocimiento locales e indígenas, incluida la convocación de talleres de diálogo global y el desarrollo. estudios de caso; emprender el trabajo para facilitar el aporte de los sistemas de conocimiento indígenas y locales, en particular para poner a prueba los procedimientos y enfoques preliminares para trabajar con los sistemas de conocimiento indígenas y locales en las evaluaciones aceleradas, temáticas, regionales y subregionales; asesorar sobre el establecimiento de una lista y una red de expertos en conocimiento indígena y local para apoyar el trabajo del Panel; apoyar el establecimiento de un mecanismo participativo para los sistemas de conocimiento indígenas y locales para facilitar los vínculos entre las comunidades indígenas y locales y los científicos; y establecer enlaces con el equipo de tareas sobre creación de capacidad y el equipo de conocimientos y datos para garantizar que aborden las cuestiones relacionadas con los conocimientos locales y culturales como contrapartida y enfoques complementarios.

La organización de eventos como las Jornadas de Etnobiología también juega un papel fundamental en la reunión de diversas áreas de conocimiento e investigadores bolivianos: biólogos, botánicos, agrónomos, arqueólogos, sociólogos, antropólogos y otros. Al presente en Bolivia hemos tenido dos versiones desde 2016 y el intercambio ha entregado interesantes temas de investigación que están en progreso. Las recomendaciones y conclusiones se presentaron en manifiestos para aumentar el nivel de divulgación a un público más amplio. Se espera que continúe con esta línea y busque diversificar la participación de los actores locales y los grupos objetivo de nuestra investigación con comunidades humanas.

Si bien algunos ejemplos desfavorables aún son conocidos por la trayectoria de los proyectos etnobiológicos, muchos esfuerzos y la mejor dedicación de reconocimiento a los conocedores y sabios de las comunidades humanas en términos de sus culturas y tradiciones se concentran en varios borradores y propuestas de códigos éticos. Estos códigos se convirtieron en herramientas que guían y tratan sustancialmente de generar un entorno de equidad y oportunidades compartidas para trabajos conjuntos en los que participan científicos y comunidades humanas; un claro ejemplo es el Código de ética para la investigación etnobiológica en América Latina (http://www.ethnoscientia.com/index.php/revista/arti cle/view/174) publicado en 2018. Al establecer espacios para la presentación previa, libre e informada del proyecto que se desarrollará con su alcance, actividades y productos, las condiciones también se abrieron y fortalecieron para establecer acuerdos de colaboración y contrapartida, entre los sabios de los indígenas y los científicos. Luego, se generaron pactos de contraparte que ilustraron la validez del proyecto en base a las contribuciones de ambas partes. Dependiendo de cada proyecto, las tareas y competencias asignadas se distribuyen de acuerdo con las capacidades y el aprendizaje de quienes forman estas relaciones de cooperación. Los compromisos y las responsabilidades también se incluyen para contribuir a las actividades $y$ productos del proyecto.

En términos prácticos, cada experiencia busca optimizar la interacción establecida en la investigación realizada con el apoyo y la colaboración de las comunidades humanas y los pueblos. Como resultados preliminares y con una amplia repercusión es que los sistemas de análisis entre científicos y comunidades están adaptados; el intercambio de conocimientos genera una opción más rica y completa. Entre los resultados más convincentes que se derivan de estos estudios colaborativos, se encuentra el punto de análisis de ambas partes: basado en el conocimiento cultural y en cómo utilizan entornos naturales versus el conocimiento científico basado en protocolos y esquemas estandarizados que sistematizan los 
datos y consolidan un escenario horizontal. Los puntos de vista también están armonizados en una fusión complementaria de ambas capacidades. Aún más importante es que se establecen nuevas y más sólidas líneas de cooperación y los proyectos de investigación se rigen por una secuencia continua de evaluaciones, que enriquecen todo el análisis, el rescate de tradiciones y las opciones de complementariedad laboral a través de talleres participativos e intercambio de información.

La orientación derivada de estas investigaciones etnobiológicas en Bolivia se suma al entorno cultural y tradicional para involucrar también a los productores y recolectores de los productos de la naturaleza, quienes no necesariamente han habitado estos espacios en capacidad o conocimiento histórico, sino más bien recientemente. Son inmigrantes nuevos en el país por menos de 10 años y no conocen esos paisajes naturales de manera adecuada. Por lo tanto, en los procesos de entrega de sus conocimientos, los resultados de ciertos estudios resultan en una guía y diferentes tipos de manuales para corregir errores, cambiar prácticas hostiles con producción sostenida y resaltar los procedimientos positivos. Hemos avanzado más y desarrollado escenarios con un amplio intercambio de conocimientos, pero seguramente todavía necesitamos establecer ventanas de trabajo colaborativo donde el conocimiento sea horizontal y complementado. Cada vez las etapas consolidan esos vínculos bajo consenso, respeto y participación conjunta. 